\title{
Evaluation of Two Procedures for Selecting the CIE Standard Sky Type Using High Dynamic Range Images
}

\author{
Ignacio García1, Marian de Blas ${ }^{1}$, José Luis Torres ${ }^{1}$, Begoña Hernández², Carlos Sáenz² and \\ Mikel Ormazábal ${ }^{1}$ \\ ${ }^{1}$ Department of Engineering, Public University of Navarre, Pamplona (Spain) \\ 2 Department of Science, Public University of Navarre, Pamplona (Spain)
}

\begin{abstract}
The characterization of sky conditions according to the CIE Standard General Sky classification requires knowledge of diffuse luminance angular distribution in the sky vault. This variable is usually measured by sky scanners. However, commercial sky scanners have different drawbacks related to their resolution and measurement time. An alternative to these devices is the use of sky images captured with a digital camera equipped with a fisheye lens. The range of luminances that may occur in the sky makes it necessary to use high dynamic range (HDR) images obtained by the fusion of a series of low dynamic range (LDR) images. Two procedures for the characterization of sky conditions according to the CIE standard using HDR images have been applied and evaluated.
\end{abstract}

Keywords: CIE standard skies, Sky imager, HDR imaging

\section{Introduction}

The optimal use of natural daylight in buildings requires an accurate knowledge of sky luminance distribution. Early research in the field of sky luminance distribution focused on overcast and clear skies modeling. This is the case of the Moon and Spencer (1942) model for overcast skies and the Kittler (1965) model for clear skies that resulted in two International Commission on Illumination (CIE) standards (CIE, 1955, 1973, respectively). However, clear and overcast skies represent only the extremes of a wide range of variability of real sky conditions. In order to deal with this reality, a second type of luminance distribution models for all sky conditions emerged, including the models developed by Perraudeau (1988), Matsuura and Iwata (1990), Perez et al. (1990), Brunger and Hooper (1993), Perez et al. (1993), Igawa et al. (2004) and Igawa (2014). For their part, Kittler et al. $(1998,1997)$ proposed a set of 15 sky standards whose luminance distributions, called Standard Sky Luminance Distributions (SSLD), were described in the SSLD Catalog. This proposal was consolidated in 2003 with the CIE Standard General Sky (CIE, 2003) that incorporated the existing CIE standard skies. Since the proposal of the CIE Standard General Sky, a number of research work has centered on the development of procedures to classify the sky from luminance measurements according to it.

The measurement of sky luminance distribution is carried out by the so-called skyscanners. Sometimes, these devices are experimental prototypes as it is the case of the one used by Perez et al. (1990) or the portable spectral sky-scanner developed by Kómar et al. (2013). However, commercial skyscanners are the most widely used. These latest devices measure the luminance corresponding to the 145 patches of sky hemisphere, according to the CIE Guide (CIE, 1994). Apart from their high cost, the use of commercial skyscanners has a number of drawbacks. Firstly, skyscanners measure luminance in a series of discrete sky positions with a certain field of view, therefore they are not able to cover the whole sky hemisphere. In addition, some of commercial models spends more than four minutes scanning the 145 sky positions. On unstable days, the sky conditions may change during this measurement period.

The use of calibrated images of the sky, in which the pixel counts are converted into a luminance level, has been positioned as a feasible alternative to sky scanners. Commercial digital cameras equipped with a fisheye lens can cover the whole sky with a higher resolution (dependent on the number of sensor pixels) than sky scanners. 
In addition, the image capture time is noticeably lower. However, the dynamic range of conventional digital cameras is not high enough to capture the whole range of luminances that can occur in the sky, especially when the sun is visible. In this regard, the use of high dynamic range (HDR) images, obtained by the combination of a number of low dynamic range (LDR) images taken with different exposures, can cover the whole range of sky luminances. There is a number of references in the literature related to the use of HDR images for determination of luminance: (Cai, 2015, 2012; Cai and Chung, 2010; Inanici, 2010, 2006).

Previous research on the use of HDR images for generating illuminance maps in Chile was conducted by Piderit et al. (2014). Souza et al. (2016) proposed a procedure for the determination of the CIE standard from sky HDR images. The indicatrix and gradation functions were extracted from a calibrated HDR image by an adaptation of the procedure described by Kovab et al. (2012), originally oriented to the characterization of sky scanner measurements.

In this work, an alternative procedure for sky characterization according to the CIE standard using HDR images is proposed. The method applied by Souza et al. (2016), called Relative Gradation and Indicatrix method (RGI) and the new proposed procedure, named Relative Zenith Luminance (RZL) have been used to characterize the skies of Pamplona (Spain) from July to October 2018. The results of RGI and RZL procedures have been compared in order to analyze the uncertainties in the classification. Likewise, the image-based standard skies have been contrasted with those obtained from skyscanner measurements.

\section{Materials}

The characterization of sky conditions according to CIE standard has been performed both from sky HDR images and from luminance distribution measurements obtained from the Public University of Navarre radiometric station (42 $47^{\prime} 32^{\prime}$ ' N, $1^{\circ} 37^{\prime} 45^{\prime}$ ' W, $435 \mathrm{~m}$ above sea level) located in Pamplona (Spain). Images were taken using a Canon EOS 6D digital camera with a CMOS full-frame sensor which has a maximum resolution of $5472 \times 3648$ pixels. The camera exhibits a particular response curve which links the luminance to the digital value of the pixels in the image. Not all luminance range can be extracted from the image, as low luminance values result in undesired noises whereas saturation takes place with high values.

The camera is equipped with a Sigma $8 \mathrm{~mm}$ F/3.5 fish-eye lens, which let obtain images with a $180^{\circ}$ field of view. The lens has been geometrically calibrated in order to accurately determine its projection function. It has been checked that the empirical lens projection fits to the theoretical equisolid projection. In addition, the vignetting effect, which produces a luminance attenuation at the edges of the image with respect to its center, has been estimated and corrected. Radiometric camera calibration has not been necessary for the characterization of the CIE standard since luminance values relative to the zenith are used in the sky classification process.

The measurement campaign was conducted between July and October 2018. A total of 1888 HDR images were obtained, each one composed of eight LDR images. Every set of LDR images have been fused using the HDRgen command from Radiance software (Ward, 2005) to obtain a HDR image. Each of these LDR image sets was taken with the same aperture (f/22) and the exposure times presented in Table 1.

Simultaneously to the capture of sky images, sky luminance distribution was measured by a skyscanner EKO MS-LR321. Three criteria have been considered for the quality control of the luminance measurements. Firstly, all records of individual sky patches exhibiting values out of the skyscanner measuring range $\left(0-50 \mathrm{kcd} \cdot \mathrm{m}^{-2}\right)$ have been discarded. Likewise, all individual scans corresponding to sky sectors whose center is closer than $6^{\circ}$ to the sun have also been discarded since the luminance and the radiance of the sun exceed the measuring range of the skyscanner's sensors by several orders of magnitude. Thirdly, all records whose integration on the horizontal plane deviates more than $30 \%$ from the measured diffuse irradiance in the same time period have been discarded.

Tab. 1: Exposure times of each image of the set.

\begin{tabular}{|l|c|c|c|c|c|c|c|c|}
\hline Image number & 1 & 2 & 3 & 4 & 5 & 6 & 7 & 8 \\
\hline Exposure time (s) & $1 / 1600$ & $1 / 800$ & $1 / 400$ & $1 / 200$ & $1 / 100$ & $1 / 50$ & $1 / 25$ & $1 / 15$ \\
\hline
\end{tabular}




\section{HDR images classification methods}

This section details two procedures for sky characterization from HDR images according the CIE standard. However, both methods have also been adapted and used for the sky classification from skyscanner measurements.

\subsection{Relative Gradation and Indicatrix (RGI) method}

This procedure, described by Kobav et al. (2012) and adapted by Souza et al. (2016), is aimed at determining the gradation and indicatrix sky functions. The CIE standard results from the combination of the obtained gradation and indicatrix functions. The process of identifying gradation and indicatrix functions from HDR images as well as of obtaining the CIE sky type by combination of both functions is described below.

a) Determination of the indicatrix group

According to the CIE and ISO standard CIE S 011/2003 (CIE, 2003), the luminance relative to zenith at a given point of the sky vault, $l(Z, \gamma)$, is given by the Eq. 1 . The indicatrix function, $f(\chi)$ expresses the relationship between the luminance at a sky point located at an angular distance from the sun $(\chi)$ and that at the point where $\chi$ is equal to $90^{\circ}$. The indicatrix function is related to the scattering of the solar radiation as it passes through the atmosphere. The gradation function, $g(Z)$ characterizes the luminance variation from the zenith $(Z=0)$ to the horizon $\left(Z=90^{\circ}\right)$.

$$
l(Z, \gamma)=\frac{L(Z, \gamma)}{L_{Z}}=\frac{f(\chi) \cdot g(Z)}{f\left(Z_{S}\right) \cdot g(0)}
$$

Considering Eq. 1, if the relative-to-zenith luminance values of the pixels belonging to a certain almucantar (constant $Z$ ) are retrieved, the quotient between such luminances in the different points of said almucantar (which will have different $\chi$ ) and that of a point of this in which $\chi=90^{\circ}$, will provide the successive points of the observed indicatrix function, according to Eq. 2.

$$
\frac{\frac{L\left(Z, \gamma_{i}\right)}{L_{Z}}}{\frac{L(Z, \gamma)_{\chi=90}}{L_{Z}}}=\frac{\frac{f\left(\chi_{i}\right) \cdot g(Z)}{f\left(Z_{S}\right) \cdot g(0)}}{\frac{f(90) \cdot g(Z)}{f\left(Z_{S}\right) \cdot g(0)}}=\frac{f\left(\chi_{i}\right)}{f(90)}=f\left(\chi_{i}\right)
$$

The existence of a point with $\chi=90^{\circ}$ in a given almucantar implies that the inequality of Eq. 3 is verified.

$$
\left|\frac{1}{\tan (Z) \cdot \tan \left(Z_{S}\right)}\right| \leq 1
$$

As an example, two almucantars are represented in Fig. 1. In the almucantar 1 there are two points where $\chi=90^{\circ}$, symmetrical to the solar meridian, whose azimuths meet the Eq. 4. In contrast, in the almucantar 2, there is no point where $\chi=90^{\circ}$ since the maximum angular distance to the sun is lower than $90^{\circ}$.

$$
\left(\gamma-\gamma_{S}\right)= \pm \cos ^{-1}\left(\frac{1}{\tan (Z) \cdot \tan \left(Z_{S}\right)}\right)
$$

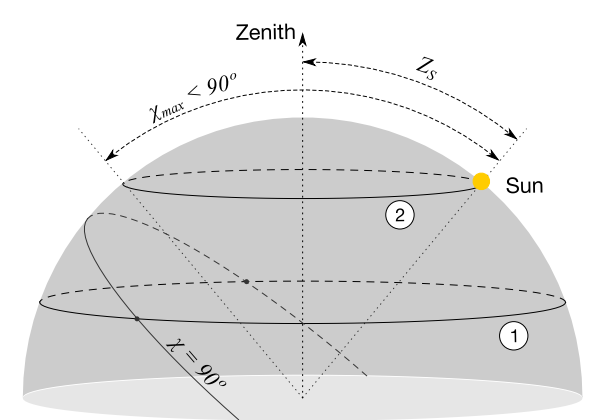

Fig. 1: In this representation of the sky vault, two almucantars are shown, denoted as 1 and 2 . Points with $\chi=90^{\circ}$ are also represented. In almucantar 1 there are two points where $\chi=90^{\circ}$. In contrast, there is no point where $\chi=90^{\circ}$ in almucantar 2 .

According to Eq. 3, in case the almucantar corresponding to the sun elevation is chosen at a given moment, there will be points with $\chi=90^{\circ}$ only when $Z_{S}$ is higher than $45^{\circ}$. In case other almucantar is chosen to identify 
the indicatrix function, it must be taken into account that, as its zenith angle moves away from $Z_{S}$, the minimum distances to the sun increase. This causes a loss of information about the indicatrix function for small values of $\chi$. In order to extend the observed indicatrix function to a wider range of $\chi$ values, which will ensure a better identification of the indicatrix group, the following criteria have been adopted in this work:

- If $Z_{S} \geq 66^{\circ}$, pixel values corresponding to the solar almucantar, where $Z=Z_{S}$, are used. This ensures that the range of $\chi$ extends from $6^{\circ}$ to $132^{\circ}$.

- If $Z_{S}<66^{\circ}$, the pixel values corresponding to two almucantars are used. The first one is the closest to the solar almucantar with $Z>Z_{S}$, where points with $\chi=90^{\circ}$ can be found. These measurements allow to define the experimental indicatrix function for small values of $\chi$. The second almucantar is that of $Z=78^{\circ}$. In this way, it is ensured, in the worst case (which corresponds to the solar noon of the summer solstice), that the experimental indicatrix function extends to values of $\chi$ ranging from 51 to $97^{\circ}$. According to the Eq. 2, there is no restriction to the joint use of the two aforementioned almucantars, provided that the luminances relative to the points where $\chi=90^{\circ}$ in each almucantar are used.

Subsequently, it is calculated the RMSD between the experimental indicatrix function values and those corresponding to each of the six standard indicatrix functions. The standard indicatrix group exhibiting the lowest RMSD is assigned to the sky under study.

b) Determination of the gradation group

The relative-to-zenith luminances of interest are those corresponding to the points with a constant distance to the sun $(\chi)$. In these points the indicatrix function remains constant and the luminance variation that can observed is only attributable to the gradation function. For a given $\chi$, these points are located in the circumference that results from the intersection of the sky vault and a cone whose vertex is in the center of said vault, its axis follows the sun vector direction and has an opening angle of $\chi$ (see Fig. 2). If an angular distance to the sun is chosen which satisfies that $\chi=Z_{S}$, the Eq. 1 is reduced to the Eq. 5. Therefore, the observed luminance relative to the zenith in the successive $Z_{i}$ provides the values of the experimental relative gradation function.

$$
\frac{L(Z, \gamma)_{\chi=Z_{S}}}{L_{Z}}=\frac{f\left(Z_{S}\right) \cdot g(Z)}{f\left(Z_{S}\right) \cdot g(0)}=\frac{g(Z)}{g(0)}
$$

a)

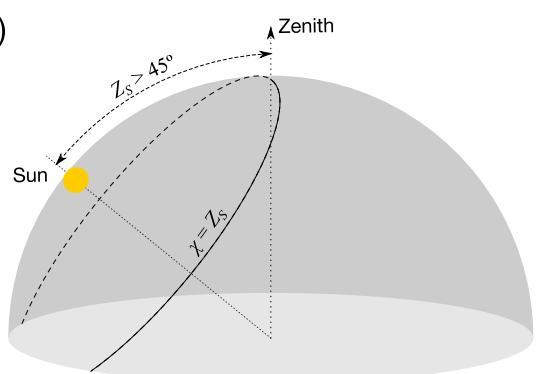

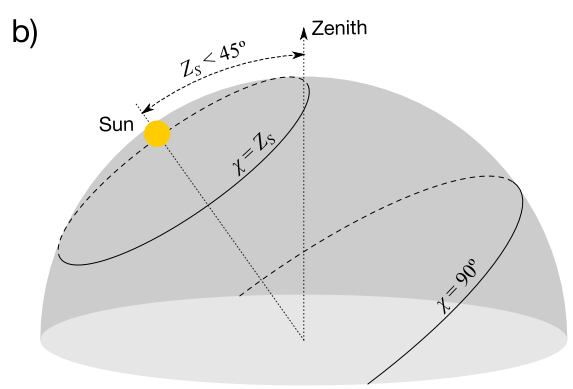

Fig. 2: When $Z_{S}>45^{\circ}$, the line defined by the points with $\chi=Z_{S}$ sweeps all sky almucantars (a). When $Z_{S}<45^{\circ}$, such line is not enough to cover the lower almucantars. Therefore, it is necessary to include the line defined by the points with $\chi=90^{\circ}\left(b^{\circ}\right)$.

It can be seen in Fig. 2 that if $Z_{S}>45^{\circ}$, when taking the luminances of the points where $\chi=Z_{S}$, the complete experimental gradation function can be described from $Z=0$ to $Z=90^{\circ}$. In contrast, if $Z_{S}<45^{\circ}$ the information from the lower almucantars is lost. In this case, the comparison among the experimental gradation function and the standard ones is less accurate. For this reason, the following criteria have been adopted in this work:

- If $Z_{S}>45^{\circ}$ the pixel values corresponding to the distance to the sun $\chi=Z_{S}$ is considered (see Fig. 2).

- If $Z_{S}<45^{\circ}$ the pixel values at two different distances to the sun are considered. The first one is equal to $Z_{S}$ and the second one is equal to $90^{\circ}$, as already proposed by Kittler (1985) (see Fig. 2). In this case, for $\chi=$ $90^{\circ}$, the Eq. 1 becomes Eq. 6. Unlike what happens when $\chi=Z_{S}$, the luminance values relative to the zenith do not provide the relative gradation function values. To obtain $f\left(Z_{S}\right)$ it is enough to divide the luminance values relative to the zenith for the same $Z$ in the two circles represented in Fig. 2, as described in Eq. 7. The successive values of the experimental gradation function are those corresponding to $\chi=Z_{S}$ plus those corresponding to $\chi=90^{\circ}$ multiplied by $f\left(Z_{s}\right)$. 


$$
\begin{aligned}
& \frac{L(Z, \gamma)_{\chi=90^{\circ}}}{L_{Z}}=\frac{f\left(90^{\circ}\right) \cdot g(Z)}{f\left(Z_{S}\right) \cdot g(0)}=\frac{g(Z)}{f\left(Z_{S}\right) \cdot g(0)} \\
& f\left(Z_{S}\right)=\frac{L(Z, \gamma)_{\chi=Z_{S}}}{L(Z, \gamma)_{\chi=90^{\circ}}}
\end{aligned}
$$

As in the case of the indicatrix function, the assigned standard gradation group is the one with the lowest RMSD in relation to the experimental values.

c) Determination of the CIE sky according to the gradation and indicatrix group

By combining the six gradation groups with the six indicatrix groups, it would be possible to identify up to 36 different sky types. However, only 15 of all possible combinations are considered as standard by the CIE. In this sense, Dumortier and Kobav (2007) proposed a reduction from 36 sky types to 15 CIE sky types. For this, 36 sky luminance distributions were calculated for 5181 sky elements for each sky type. Non-standardized sky luminance distributions were compared with each of the standard skies. The sky type with the lowest RMSD was considered the equivalent CIE sky type. The results of this analysis were specified in a single reduction table suitable for any solar elevation. This proposal was used by Kobav et al. (2012) for the characterization, according to the CIE standard, of sky scanner measurements made at the International Daylight Measurement Program station in Lyon (France). This solution has been analyzed in the present work and it has been observed that there is a dependence of the combinations with solar elevation. Therefore, the new combinations obtained according to the sun elevation have been used.

Standard gradation and indicatrix profiles are represented in Fig. 3a y Fig. 3b respectively. By way of example, the relative luminance values extracted from an HDR image are also represented in red. As a result of the comparison of each luminances series with the standard profiles, as described in the previous sections, a gradation group $\mathrm{V}$ and an indicatrix group 4 are obtained. The combination of both functions results in a standard sky type 12, that is, "CIE standard clear sky, low luminance turbidity" as defined by CIE (2003).
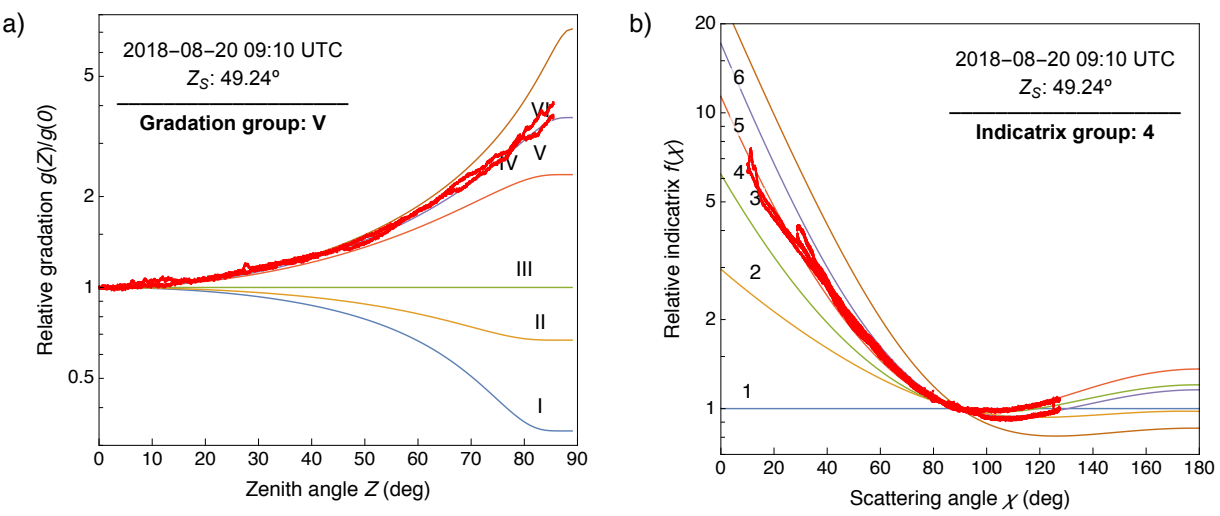

Fig. 3: Standard gradation profiles and relative gradation values (in red) extracted from an HDR image (a). Standard indicatrix profiles (b) and relative indicatrix values (in red) extracted from an HDR image.

In both Fig. 3a and 3b, two sets of data can be seen with a slightly differentiated trend. Each of these trends corresponds to the relative luminance values corresponding to each side of the solar meridian. This indicates that the sky luminance distribution is not strictly symmetric in this case.

\subsection{Relative Zenith Luminances (RZL) method}

This procedure is an adaptation of the method proposed by Tregenza (2004) and used in the characterization of CIE standard skies in Pamplona from skyscanner measurements (Torres et al., 2010a, 2010b). In the original procedure, the CIE standard was determined by comparing the measured relative luminance distribution (in 145 sky patches) with the luminance distribution corresponding to each of the 15 standard skies. In the present study, this analysis has been applied to each pixel in the image. However, whereas in the original procedure the luminance of each area of the sky is related to the diffuse horizontal illuminance (obtained by the integration of the measured luminances), in this case the zenith luminance has been used as a reference. This change of reference can be a problem when skies in low latitudes are classified, because the zenith luminance can be influenced by the sun at certain times. However, this is not a problem in mid-latitudes locations like Pamplona 
where the sun reaches a maximum elevation of $71^{\circ}$.

The new proposed method comprises the following steps:

- Definition of a grid within which the projection of the sky vault on the horizontal plane is inscribed. The resolution of the grid must match that of the images used to characterize the state of the sky. So, in this work a grid of $5472 \times 3648$ cells is used.

- Determination of the zenith angle $(Z)$ and azimuth $(\gamma)$ corresponding to the center of each of the grid cells assuming an equisolid projection. In this way, it is possible to generate an image (of 5472 x 3648 pixels in this particular case) whose pixels are defined by the zenith angle and the azimuth of its center. However, only $9.023 \cdot 10^{6}$ pixels out of the $19.962 \cdot 10^{6}$ pixels that make up the image correspond to the sky projection. Eq. 8 relates the projected distance between a pixel and the center of the image $(R)$ and the corresponding zenith angle $(Z)$ according to an equisolid projection.

$$
R=2 \cdot \sin (Z / 2)
$$

- At a given time, when the sun position is defined by its zenith angle $\left(Z_{S}\right)$ and azimuth $\left(\gamma_{S}\right)$, an angular distribution image of luminance relative to the zenith for each of the 15 CIE standard skies is generated. To do this, the Eq. 1 is applied to each of the pixels of the image. Fig. 4 shows an example of the obtained distributions for each of the $15 \mathrm{CIE}$ standard considering a solar zenith angle of $60^{\circ}$ at solar noon.
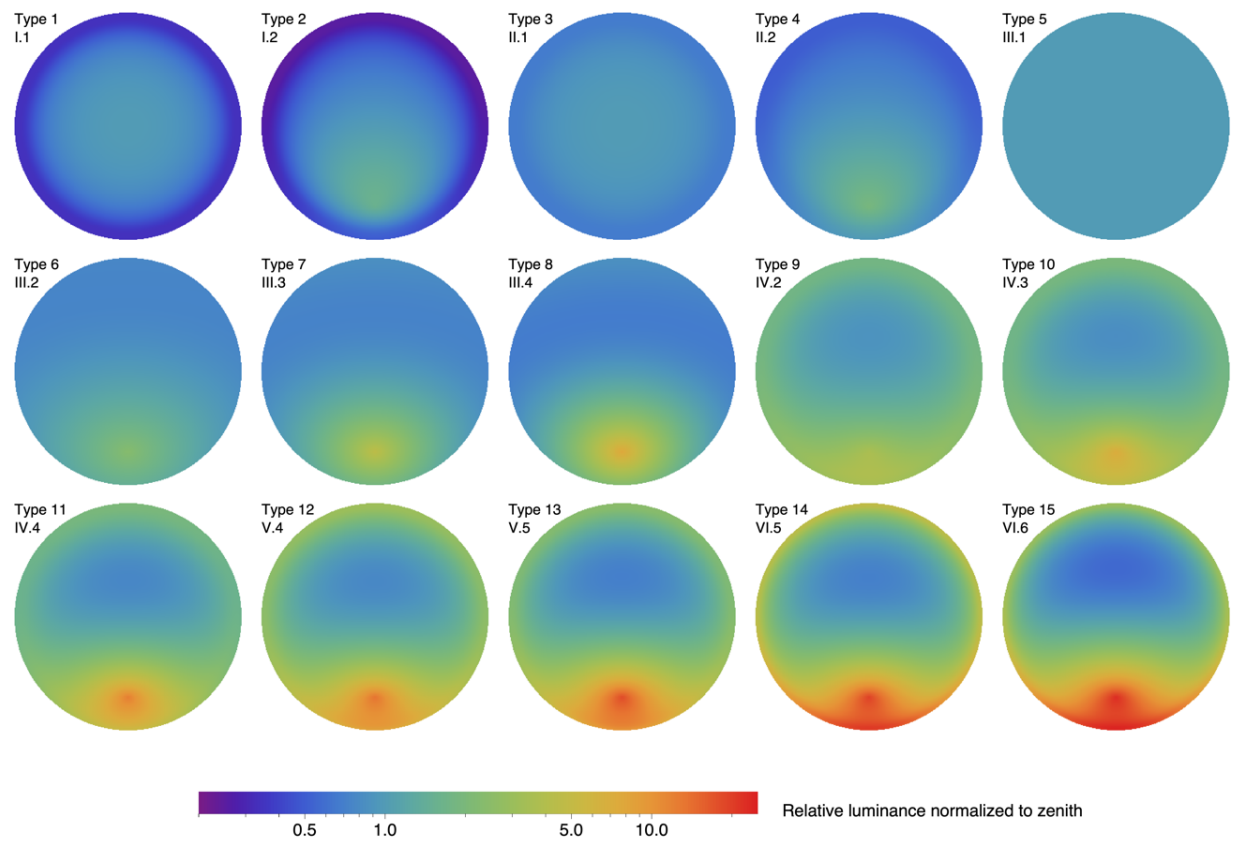

Relative luminance normalized to zenith

Fig. 4: Angular distribution of sky luminances for each of the 15 standard CIE sky types.

- Comparison of HDR image pixel values of luminance relative to zenith $\left(l_{p}\right)$ with the modeled values corresponding to each of the 15 standard skies $\left(l_{p, s t}\right)$. To do so, the RMSD is calculated using Eq. 9.

$$
R M S D_{s t}=\sqrt{\frac{1}{n_{p}} \sum_{n_{p}}\left(l_{p}-l_{p, s t}\right)^{2}}
$$

- The selected standard sky type will be the one out of the fifteen calculated in the previous step that exhibits the lowest $R M S D_{s t}$ value.

\section{Results}

Fig. 5a shows the frequencies of each CIE standard obtained from HDR images by the two procedures described. In both cases, a higher incidence of type 12 skies (V.4) can be observed. However, there are noticeable differences between the results obtained by both procedures. Fig. $5 \mathrm{~b}$ represents the coincidence frequencies of the CIE types obtained by RGI and RZL methods. 
a)

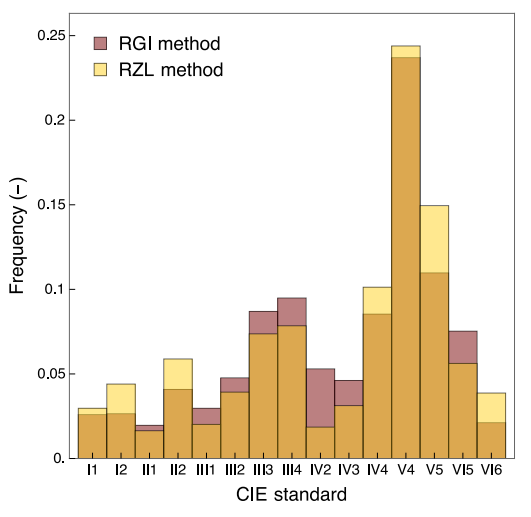

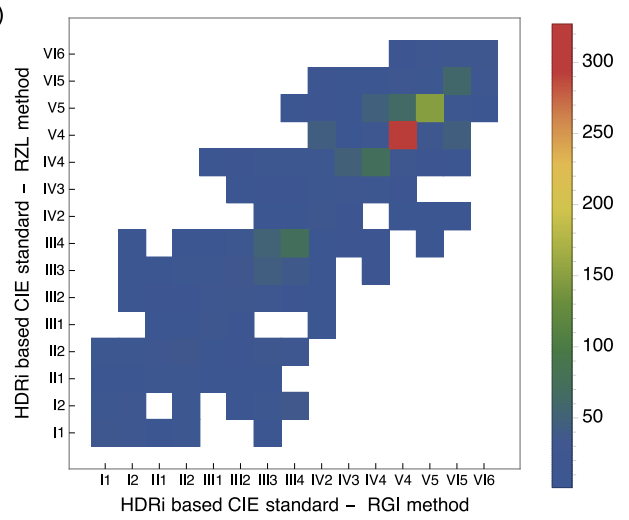

Fig. 5: CIE standard sky type occurrence frequencies obtained by RGI and RZL methods from HDR images (a). Coincidence matrix of standard sky types obtained by RGI and RZL methods from HDR images (b), the colored scale corresponds to the number of cases with equal resulting sky type.

A detailed analysis of these results reveals that the classification by both procedures coincides in $49.26 \%$ of the skies, while in $25.34 \%$ of the cases there is a difference of one CIE standard (see Fig. 6a). As explained in Section 4.2, 15 RMSD values are calculated when determining the standard sky type using the RZL method. In this way, it is possible to order the 15 standard skies from the lowest to the highest RMSD value, so that the one that placed the first in the ordered list is selected. In many cases, the sky standard type which occupies the second place in the classification has an RMSD value close to the first one. For this reason, a new comparison of the sky types determined by both procedures has been made considering together the two sky types that occupy the first positions according to the RZL method (see Fig. 6b). As it can be seen, in this case the percentage of coincidence raises up to $73.01 \%$.

a)

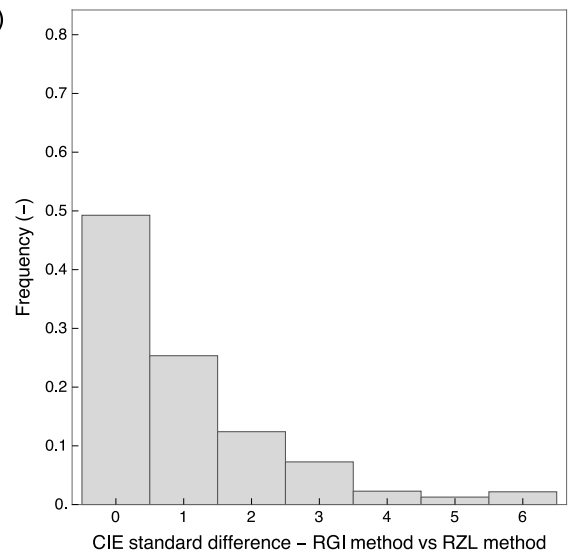

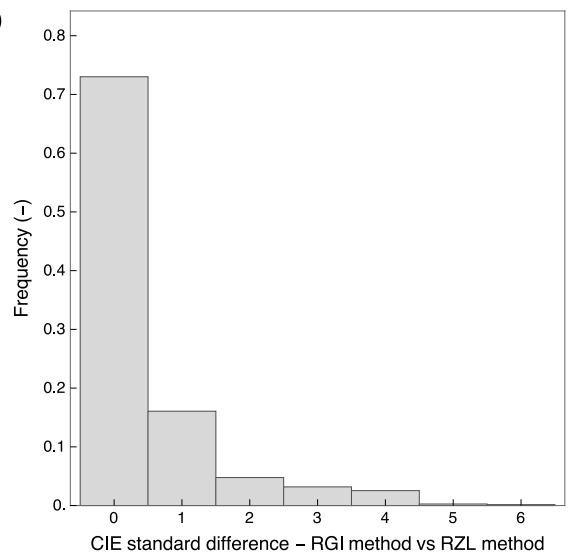

Fig. 6: Frequency distribution of differences, in absolute value, among the sky types obtained by the RGI and RZL methods from HDR images (a). Frequency distribution of differences among the sky types obtained by the RGI and RZL methods from HDR images when considering together the two sky types that occupy the first positions according to the RZL method (b).

The classifications obtained from HDR images by means of the two procedures described have been compared against those obtained by applying both procedures to sky luminance distribution measurements made by a skyscanner. Fig. 7a compares the resulting sky type frequencies with the RGI procedure when using HDR images and the simultaneously obtained skycanner measurements. It can be seen that there are significant differences in some cases, especially in the the standard sky type 12 (V4) frequency, which is reduced more than $50 \%$ when using the skyscanner measurements. Fig. $7 \mathrm{~b}$ shows the coincidence frequencies of the CIE types obtained by the RGI method from HDR images and from skyscanner measurements. Although some dispersion takes place, the highest frequencies are grouped around the diagonal of the graph. 
a)

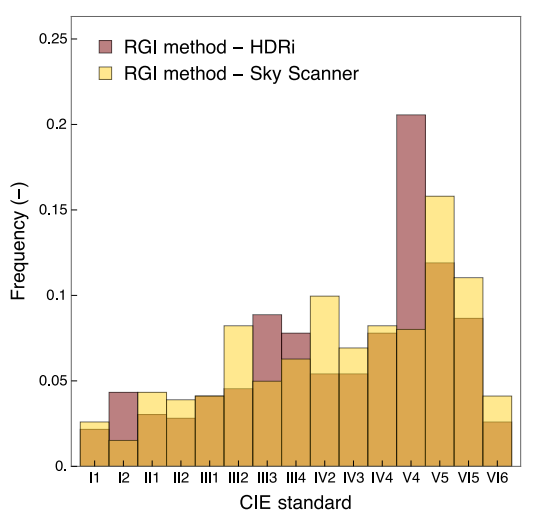

b)

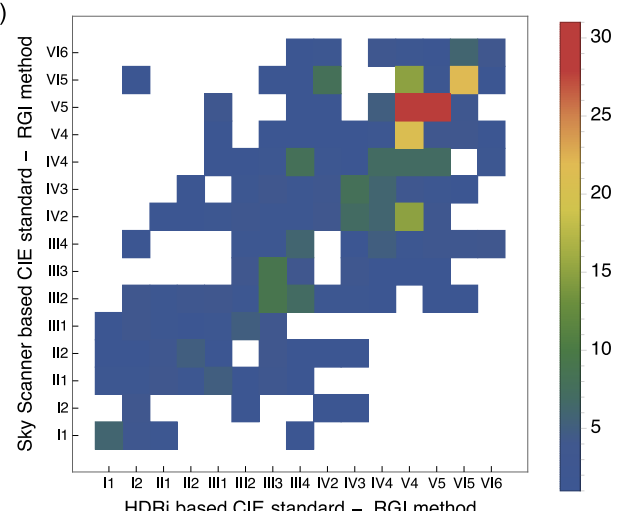

Fig. 7: CIE standard sky type occurrence frequencies obtained by RGI method from HDR images and from skyscanner measurements (a). Coincidence matrix of standard sky types obtained by RGI method from HDR images and from skyscanner measurements (b), the colored scale corresponds to the number of cases with equal resulting sky type.

The differences obtained when characterizing the skies by means of the RGI method from HDR images and from skyscanner measurements are represented in Fig 8. In this case, the standard sky type matches become the $29.22 \%$ of the total records whereas in $24.46 \%$ of the cases there is a difference of one type of sky between the two classification methods.

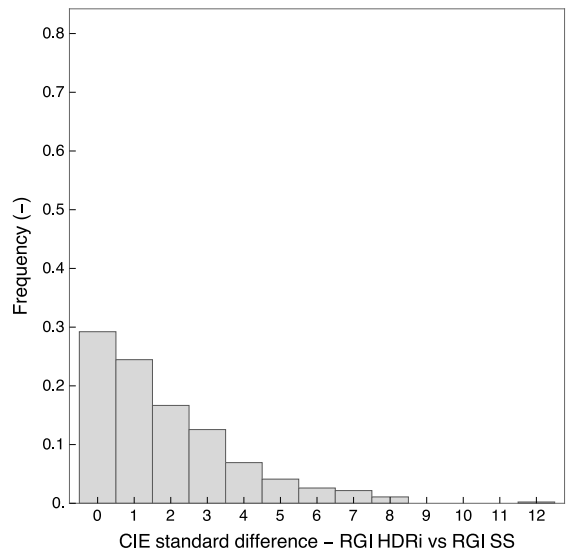

Fig. 8: Frequency distribution of differences, in absolute value, among the sky types obtained by RGI method from HDR images and from skyscanner measurements.

Fig. 9a shows the standard sky type frequencies obtained by means of the RZL method when using HDR images and skyscanner measurements. Again, considerable differences between both classifications are appreciated. The proportion of type 15 skies (VI6) is significantly increased when using the measurements of the sky scanner to the detriment of the sky types 12 (V4) and 13 (V5), which undergo a marked reduction. These differences can be seen in detail in Fig. 9b.

A possible reason for the observed discrepancies, especially in clear skies (11 to 15), may be a poor definition of the indicatrix function as a result of the necessary discarding of the skyscanner measurements from the sky patches closest to the sun. This can lead to uncertainties and differences when discriminating among the five clear sky types at a specific time. In this regard, the use of hemispherical HDR images, which are able to capture the full range of the sun and the sky luminances, is an advantage when determining the sky type in comparison to the use of skyscanner measurements. However, this issue requires further analysis that will be addressed in future research. 
a)

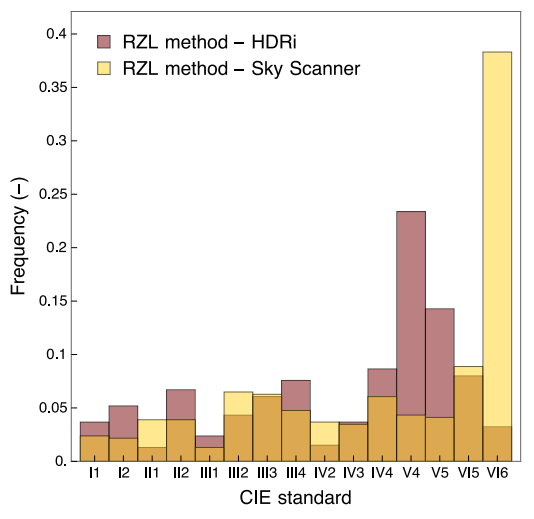

b)

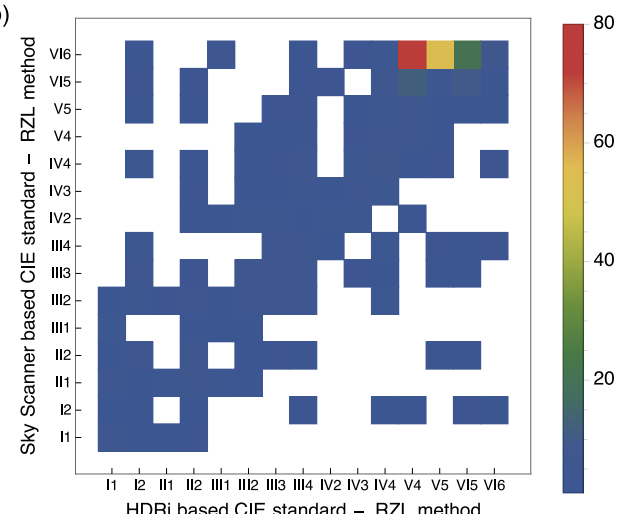

Fig. 9: CIE standard sky type occurrence frequencies obtained by RZL method from HDR images and skyscanner measurements (a). Coincidence matrix of standard sky types obtained by RZL method from HDR images and skyscanner measurements (b), the colored scale corresponds to the number of cases with equal resulting sky type.

As Fig. 10a shows, the proportion of coincidences among the sky classifications obtained with the RZL method from HDR images and from skyscanner measurements is very low (16.88\%). Again, as in the case of Fig. 6b, a comparison has been made between both classifications considering together the two sky types with the lowest RMSD (see Fig. 10b). In this case, the frequency of coincidences increases up to $49.57 \%$.

a)

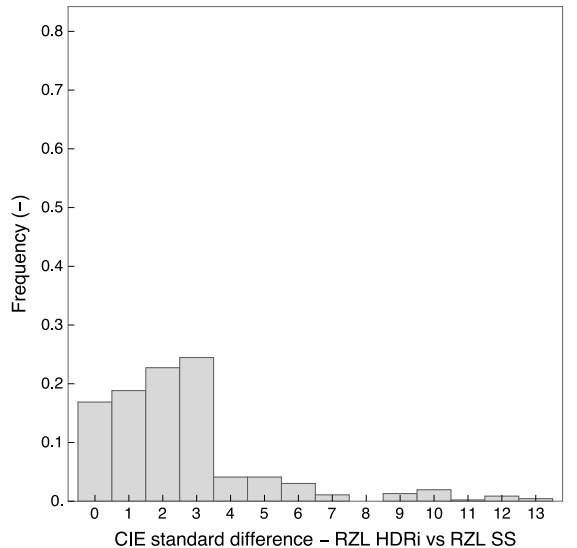

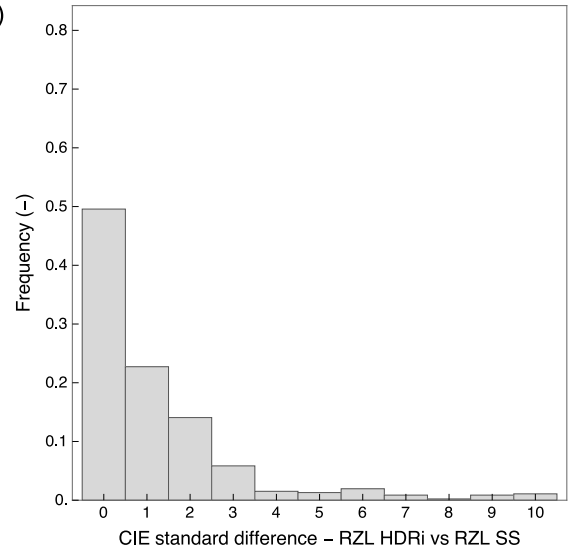

Fig. 10: Frequency distribution of differences, in absolute value, among the sky types obtained by the RZL method from HDR images and from skyscanner measurements (a). Frequency distribution of differences among the sky types obtained by the RZL method from HDR images and from skyscanner measurements when considering together the two sky types located in the first positions according to the RZL method (b).

\section{Conclusions}

Two procedures for the characterization of sky conditions according to the CIE standard using HDR hemispherical sky images have been applied and evaluated in this study. The obtained sky classification by means of HDR images when applying the Relative Gradation and Indicatrix method (RGI) and the proposed Relative Zenith Luminance method (RZL) shows a percentage of coincidences of $50 \%$. When the two more probable resulting sky types with each method are compared the percentage of coincidences raises up to $73 \%$.

Likewise, the classifications obtained from HDR images by means of the two procedures described have been compared against those obtained by applying both procedures to sky luminance distribution measurements made by a skyscanner. The use of HDR images for sky type classification offers promising results if compared to skyscanner measurements, as the sky area closest to the sun is well defined and images are taken in a very short period of time, which overcome the problems of the need of discarding the sky patches closest to the sun because of saturation as well as of the risk of variable sky conditions due to the measuring time the skyscanner need. 


\section{Acknowledgments}

This work has been performed as a part of Project RECHICRAL (0011-1365-2017-000168), co-financed by the Government of Navarre and the European Regional Development Fund through the FEDER Operational Program 2014-2020 of Navarre, and Project IRILURREFLEX (ENE2017-86974-R), financed by the Spanish State Research Agency (Agencia Estatal de Investigación, AEI) and European Regional Development Fund (Fondo Europeo de Desarrollo Regional, FEDER).

\section{References}

Brunger, A.P., Hooper, F.C., 1993. Anisotropic sky radiance model based on narrow field of view measurements of shortwave radiance. Sol. Energy 51, 53-64. https://doi.org/10.1016/0038-092X(93)90042-M

Cai, H., 2015. Using High Dynamic Range Photogrammetry for Luminance Mapping of the Sky and the Sun, in: AEI 2015. American Society of Civil Engineers, Milwaukee, WI, pp. 316-330. https://doi.org/10.1061/9780784479070.029

Cai, H., 2012. High dynamic range photogrammetry for synchronous luminance and geometry measurement. Light. Res. Technol. 45, 230-257. https://doi.org/10.1177/1477153512453273

Cai, H., Chung, T., 2010. Improving the quality of high dynamic range images. Light. Res. Technol. 43, 87102. https://doi.org/10.1177/1477153510371356

CIE, 2003. Spatial Distribution of Daylight - CIE Standard General Sky, Standard CIE S 011/E:2003/ISO 15469:2004.

CIE, 1994. Guide to recommended practice of daylight measurement, CIE publication 108. Vienna, Austria.

CIE, 1973. Standardization of luminance distribution on clear skies, Pub. CIE No.22, TC-4.2.

CIE, 1955. Natural Daylight. Official Recommendation, Compte Rendu, CIE 13th Session, Committee E-3.2, vol. II, parts 3-2, II-IV\&35-37.

Dumortier, D., Kobav, M.B., 2007. Deriving CIE sky types from horizontal irradiances, in: Proceedings of 26th Session of the CIE (Volume 1). Commission Internationale de L'Eclairage, Beijing.

Igawa, N., 2014. Improving the All Sky Model for the luminance and radiance distributions of the sky. Sol. Energy 105, 354-372. https://doi.org/10.1016/j.solener.2014.03.020

Igawa, N., Koga, Y., Matsuzawa, T., Nakamura, H., 2004. Models of sky radiance distribution and sky luminance distribution. Sol. Energy 77, 137-157. https://doi.org/10.1016/j.solener.2004.04.016

Inanici, M., 2010. Evalution of high dynamic range image-based sky models in lighting simulation. LEUKOS J. Illum. Eng. Soc. North Am. 7, 69-84. https://doi.org/10.1582/LEUKOS.2010.07.02001

Inanici, M., 2006. Evaluation of high dynamic range photography as a luminance data acquisition system. Light. Res. Technol. 38, 123-136. https://doi.org/10.1191/1365782806li164oa

Kittler, R., 1985. Luminance distribution characteristics of homogeneous skies: A measurement and prediction strategy. Light. Res. Technol. https://doi.org/10.1177/14771535850170040301

Kittler, R., 1965. Standardisation of outdoor conditions for the calculation of daylight factor with clear skies, in: Proceedings of the CIE Intersessional Conference on Sunlight in Buildings. Newcastle upon Tyne, pp. $273-285$.

Kittler, R., Perez, R., Darula, S., 1998. A set of standard skies characterizing daylight conditions for computer and energy conscious design. Am. - Slovak grant Proj. US - SK 9205292.

https://doi.org/10.13140/RG.2.1.4798.7048

Kittler, R., Perez, R., Darula, S., 1997. A new generation of sky standards, in: Lux Europa. Proceedings of the Eight European Lighting Conference. Amsterdam, pp. 359-373.

Kobav, M.B., Bizjak, G., Dumortier, D., 2012. Characterization of sky scanner measurements based on CIE and ISO standard CIE S 011/2003. Light. Res. Technol. 45, 504-512. https://doi.org/10.1177/1477153512458916 
Kómar, L., Rusnák, A., Dubnička, R., 2013. Analysis of diffuse irradiance from two parts of sky vault divided by solar meridian using portable spectral sky-scanner. Sol. Energy 96, 1-9.

https://doi.org/10.1016/J.SOLENER.2013.07.003

Matsuura, K., Iwata, T., 1990. A model of daylight source for the daylight illuminance calculations on the all weather conditions, in: Proceedings of 3rd International Daylighting Conference. Moscow, Russia.

Moon, P., Spencer, D.E., 1942. Illumination from a non-uniform sky. Trans. Illum. Eng. Soc. 37, 707-726.

Perez, R., Ineichen, P., Seals, R., Michalsky, J.J., Stewart, R., 1990. Modeling daylight availability and irradiance components from direct and global irradiance. Sol. Energy 44, 271-289.

https://doi.org/10.1016/0038-092X(90)90055-H

Perez, R., Seals, R., Michalsky, J.J., 1993. All-weather model for sky luminance distribution-Preliminary configuration and validation. Sol. Energy 50, 235-245. https://doi.org/10.1016/0038-092X(93)90017-I

Perraudeau, M., 1988. Luminance models, in: National Lighting Conference and Daylighting Colloquium. Cambridge, UK, pp. 291-292.

Piderit, Maria Beatriz; Diaz, M; Cauwerts, C., 2014. Definition of CIE Stardard Skies and application of high dynamic range imaging technique to characterize the spacial distribution of daylight in Chile. J. Constr. 13, 2230 .

Souza, D., Scarazzato, P., Pedrini, H., 2016. Classifying skies from images: A multidimensional approach to detecting high dynamic range imaging attributes. Light. Res. Technol. 48, 559-572.

https://doi.org/10.1177/1477153516637231

Torres, J.L., de Blas, M., García, A., Gracia, A.M., de Francisco, A., 2010a. Sky luminance distribution in Pamplona (Spain) during the summer period. J. Atmos. Solar-Terrestrial Phys. 72, 382-388.

https://doi.org/10.1016/j.jastp.2009.12.005

Torres, J.L., de Blas, M., García, A., Gracia, A.M., de Francisco, A., 2010b. Sky luminance distribution in the North of Iberian Peninsula during winter. J. Atmos. Solar-Terrestrial Phys. 72, 1147-1154. https://doi.org/10.1016/j.jastp.2010.07.001

Tregenza, P.R., 2004. Analysing sky luminance scans to obtain frequency distributions of CIE Standard General Skies. Light. Res. Technol. 36, 271-281. https://doi.org/10.1191/1477153504li117oa

Ward, G.J., 2005. Anyhere Software. HDRgen. URL http://www.anyhere.com (accessed 3.1.19). 